\title{
Mecanismos de Modulación Central del Dolor: Revisión de la Literatura
}

\author{
Mechanisms of Central Pain Modulation: Literature Review
}

\author{
Schilin Wen ${ }^{1,2}$; Javiera Muñoz ${ }^{3}$; Marcelo Mancilla ${ }^{3}$; Thomas Bornhardt ${ }^{1,3,4}$; Andrés Riveros ${ }^{2,5}$ \& Verónica Iturriaga ${ }^{1,3,4}$
}

WEN, S.; MUÑOZ, J.; MANCILla, M.; BORNHARDT, T.; RIVEROS, A. \& ITURRIAGA, V. Mecanismos de modulación central del dolor: Revisión de la literatura. Int. J. Morphol., 38(6):1803-1809, 2020.

RESUMEN: La percepción del dolor resulta de múltiples y dinámicos mecanismos en el sistema nervioso central (SNC) y periférico que inhiben o facilitan el estímulo y respuesta nociceptiva. Sin embargo, la principal capacidad de modulación esta a cargo del SNC. Los estímulos nociceptivos son detectados por terminaciones nerviosas libres de neuronas periféricas que sinaptan con neuronas aferentes secundarias de la médula espinal. Luego estas fibras decusan para formar las vías nociceptivas ascendentes. Una vez alcanzadas las estructuras subcorticales, se activan las neuronas del tálamo, quienes envían el estímulo hacia la corteza somatosensorial, desencadenando la percepción consciente del dolor y activando el sistema inhibitorio descendente. Para que la modulación nociceptiva se realice, es necesaria la participación de diversas sustancias o neurotransmisores que conectan áreas del SNC especializadas. Por lo tanto, el objetivo de este estudio fue realizar una revisión de la literatura respecto de los mecanismos que participan en los procesos de modulación central del dolor.

PALABRAS CLAVE: Dolor; Dolor Nociceptivo; Dolor crónico; Neurofisiología; Neuroanatomía; Inhibición Neuronal.

\section{INTRODUCCIÓN}

La percepción del dolor es el resultado de múltiples y dinámicos mecanismos pertenecientes al sistema nervioso central (SNC) y periférico (SNP) que inhiben o facilitan el estímulo y respuesta nociceptiva. Desde que Head y Holmes, postularon la existencia de un sistema corticotálamico inhibitorio del dolor en 1911, pasando por el control supraespinal propuesto por Hagbarth y Kerr en 1954, llegando a la clásica "teoría de la compuerta" de Melzack y Wall en 1965, se han propuesto múltiples teorías para explicar los mecanismos que el organismo activa con fin de inhibir o potenciar los estímulos dolorosos (Arbaiza, 2005). La importancia de estos mecanismos recae en la experiencia dolorosa, la cual depende de la modulación dinámica de los circuitos internos del sistema nervioso. Lo anterior contribuye a comprender las diferencias observadas entre individuos en cuanto a su experiencia dolorosa (Chichorro et al., 2017).

La modulación nociceptiva es el proceso por el cual la señal dolorosa puede ser modificada a lo largo del sistema nervioso, permitiendo el control facilitador o nociceptivo y el control inhibitorio o anti-nociceptivo. La modulación inhibitoria es de carácter centrífugo y descendente, presentando entre 100 a 1000 neuronas más que la modulación facilitadora. Su función altamente coordinada permite al sistema un equilibrio entre los distintos estímulos percibidos.

La modulación del dolor inicia en el SNP a nivel de las terminaciones nerviosas libres, sin embargo, la principal capacidad de modulación se va a encontrar en el SNC. Por lo tanto, el objetivo de este estudio fue realizar una revisión de la literatura respecto de los mecanismos que participan en la neuromodulación central del dolor.

\section{MATERIAL Y MÉTODO}

Se realizó una búsqueda en las bases de datos PubMed, Scopus y Web of Science. En la fase inicial, se identificaron los términos de búsqueda, incluyendo los tér-

\footnotetext{
${ }^{1}$ Sleep \& Pain Research Group, Universidad de La Frontera, Temuco, Chile.

${ }^{2}$ Doctorado en Ciencias Morfológicas, Universidad de La Frontera, Temuco, Chile.

${ }^{3}$ Especialidad en Trastornos Temporomandibulares y Dolor Orofacial, Universidad de La Frontera, Temuco, Chile.

${ }^{4}$ Departamento de Odontología Integral Adulto, Facultad de Odontología, Universidad de La Frontera, Temuco, Chile.

${ }^{5}$ Departamento de Ciencias Morfológicas, Facultad de Medicina y Ciencia, Universidad San Sebastián, Lientur 1457, Concepción 4080871, Chile.
} 
minos libres "pain modulation", "pain inhibition", "pain facilitation", "diffuse noxious inhibitory control" y "conditioned pain modulation". Posteriormente, se realizó una búsqueda sensible agregando los operadores booleanos "OR" y "AND". La búsqueda se realizó en junio de 2020, y se adaptó a cada base de datos y su idioma correspondiente. Las estrategias de búsqueda para cada base de datos consultada se detallan en la Tabla I. Los criterios de inclusión fueron: 1) artículos en idioma inglés y español; 2) artículos que tuvieran como objetivo el estudio de la modulación del dolor. Se excluyeron cartas al editor, trabajos presentados en congresos, reportes de casos y series de casos. Todas las referencias identificadas fueron extraídas a través del gestor de referencias Mendeley ${ }^{\circledR}$ para facilitar su manejo, eliminando los artículos duplicados. Los artículos fueron examinados de forma independiente por dos investigadores (JM - MM). Cada uno de los artículos fue evaluado de manera secuencial primero por título, luego resumen y finalmente el artículo completo, identificando los criterios de inclusión. Los mismos dos autores realizaron la extracción de datos de cada uno de los artículos.

\section{RESULTADOS Y DISCUSIÓN}

La búsqueda arrojó 1852 artículos en total. Posterior a la eliminación de duplicados se obtuvieron 827 artículos, de los cuales finalmente 26 artículos fueron seleccionados para la revisión. En la Tabla I se detallan los resultados obtenidos por cada base de datos.

\section{Generalidades de la neuromodulación del dolor.}

Los estímulos nociceptivos son detectados por terminaciones nerviosas libres de neuronas periféricas de tipo $\mathrm{A} \delta$ y $\mathrm{C}$, cuyos somas se ubican en los ganglios de las raíces dorsales de los nervios espinales o en ganglios sensitivos somáticos de nervios craneales. Las prolongaciones axonales de estos somas se extienden para sinaptar con las neuronas aferentes secundarias ubicadas en el cuerno posterior de la médula espinal (CPME) o en los núcleos sensitivos de los nervios craneales, respectivamente (Perena et al., 2000;
Zegarra, 2007) (Fig. 1). Las fibras originadas de los núcleos del CPME decusan para formar las vías nociceptivas ascendentes que corresponden a los tractos espinorreticular y espinotalámico; este último constituye el fascículo más importante para el ascenso de las señales nociceptivas (Zegarra; Pedrajas \& Molino, 2008). Por su parte, las neuronas pertenecientes a los nervios craneales, principalmente trigeminales, se unen a la parte interna de la vía espinotalámica en su camino ascendente, conformando la vía trigéminotalámica. Una vez alcanzadas las estructuras subcorticales, se activan las neuronas de tercer orden ubicadas en el tálamo, quienes envían el estímulo hacia la corteza somatosensorial, desencadenando la percepción consciente del dolor, las actividades subconscientes y las respuestas neuromoduladoras efectoras, endocrinas y emocionales (Zegarra; Okeson, 2013).

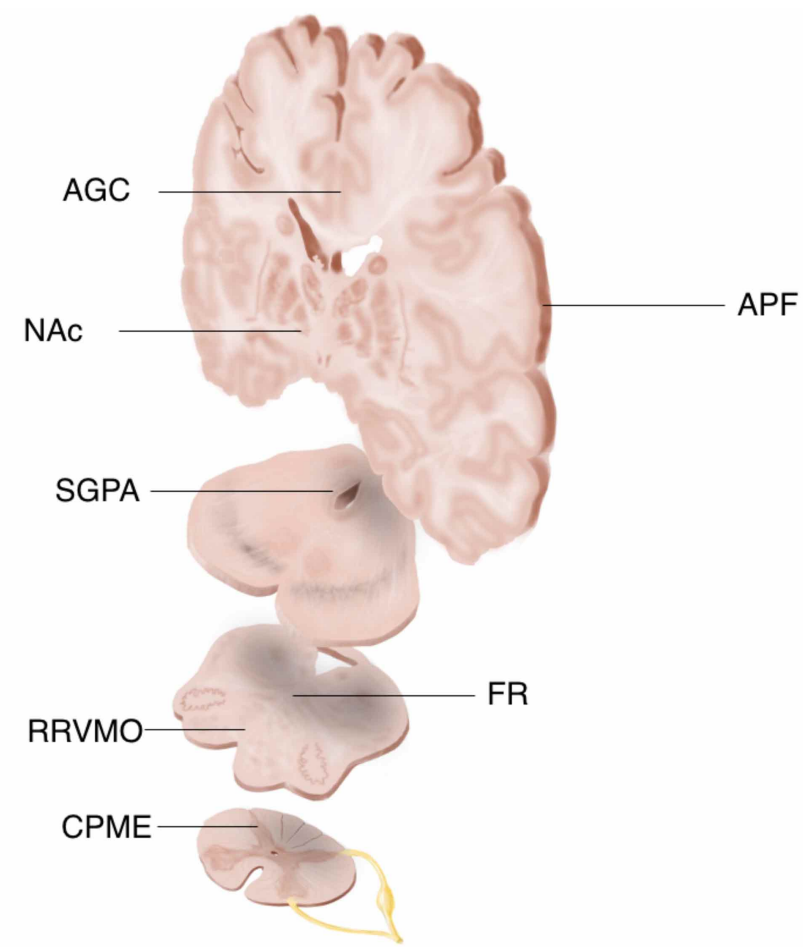

Fig. 1. AGC: Área anterior del giro cingulado, APF: Área prefrontal, NAc: Núcleo accumbens, SGPA: Sustancia gris periacueductal, FR: Formación reticular, RRVMO: Región rostral ventromedial de la médula oblongada, CPME: Cuerno posterior médula espinal.

Tabla I. Estrategias de búsqueda y resultados por cada base de datos consultada.

\begin{tabular}{clc}
\hline $\begin{array}{c}\text { Base de } \\
\text { datos }\end{array}$ & Estrategia de búsqueda & $\begin{array}{c}\text { Result ados } \\
\text { obtenidos }\end{array}$ \\
\hline PubMed & ("pain modulation" OR "pain inhibition" OR "pain facilitation") AND ("diffuse noxious & 589 \\
& inhibitory control" OR "conditioned pain modulation") & 611 \\
Scopus & ("pain modulation" OR "pain inhibition" OR "pain facilitation") AND ("diffuse noxious \\
& inhibitory control" OR "conditioned pain modulation") \\
WoS & ("pain modulation" OR "pain inhibition" OR "pain facilitation") AND ("diffuse noxious \\
& inhibitory control" OR "conditioned pain modulation") & 652 \\
\hline
\end{tabular}


De esta manera la modulación de la información nociceptiva en el SNC puede ocurrir a lo largo de todo el recorrido del impulso nervioso. Sin embargo, se han propuesto mecanismos endógenos especializados, dentro de los cuales encontramos: 1) Mecanismos corticales, 2) Mecanismos subcorticales, 3) Modulación del CPME y 4) Modulación condicionada de dolor (MCD), más conocido como CPM por sus siglas en inglés conditioned pain modulation o como diffuse noxious inhibitory control (DNIC). Estos mecanismos funcionan a través de sistemas de señalización, en los cuales participan activamente diversas moléculas neuromoduladoras.

\section{Sustancias neuromoduladoras del dolor}

a) Modulación del dolor mediada por opioides endógenos. Dentro de los opioides endógenos encontramos las encefalinas, $\beta$-endorfinas, dinorfina, endomorfinas (I y II) y la nociceptina (Orfanina FQ). Estas sustancias son liberadas por activación del sistema descendente alrededor de la sustancia gris periacueductal (SGPA), rodeando el acueducto mesencefálico (Perena et al; Arbaiza; Zegarra) (Fig. 1). Su acción se basa en el cierre de canales de $\mathrm{Ca}++\mathrm{y}$ apertura indirecta de los canales de $\mathrm{K}+$. Esto genera una hiperpolarización de los nociceptores y bloqueo de la liberación de neurotransmisores como glutamato y sustancia $\mathrm{P}$. El resultado de esto es la analgesia y disminución de la actividad del nociceptor. La efectividad de los sistemas opioides a múltiples niveles se explica por la amplia distribución de los receptores opioides en distintas áreas del SNC. De este modo, mientras que la activación de cualquiera de los receptores opioides a nivel espinal produce un efecto analgésico, a nivel supraespinal el efecto de la unión al ligando dependerá de su localización (Arbaiza).

b)Modulación del dolor mediada por Acetilcolina. La Acetilcolina $(\mathrm{ACh})$ actúa como un neurotransmisor y neuromodulador liberado desde interneuronas y proyecciones colinérgicas específicas, tanto en el cerebro como a nivel medular (Naser \& Kuner, 2018). Existen dos tipos de receptores que responden a ACh: los muscarínicos (mAChR) y nicotínicos (nAChR), cuyos mecanismos de acción definen diferencias en términos de modulación (Naser \& Kuner) (Tabla II).

Tabla II. Sustancias moduladoras del dolor, sus receptores y mecanismos de acción. SNC: sistema nervioso central; SNP: sistema nervioso periférico; RRVMO: región rostral ventromedial de la médula oblongada; mAChR: Receptores muscarínicos de acetilcolina; nAChR: Receptores nicotínicos de acetilcolina; CPME: cuerno posterior de la médula espinal; GABA: Ácido gamma aminobutírico; ME: médula espinal; 5-HT: 5-hidroxitriptamina; K+: potasio; NMDA: ácido N-metil-D-aspártico.

Sustancias Receptores Mecanismo de acción

Neuromoduladoras

Opioides endógenos $\quad \mu, \partial, \mathrm{K}$

Encefalinas: Son secretadas en la adenohipófisis y actúa a nivel hipotalámico regulando la respuesta autonómica al dolor, mientras que en el sistema límbico esta se encarga de aquellos aspectos ligados a la conducta y el estado de ánimo frente al dolor.

B Endorfinas: Se distribuyen ampliamente en el SNC y SNP. Su afinidad por receptores se asemeja a las endorfinas, pero su acción es 100 veces más potente.

Nociceptina: Inhiben los tres sistemas neuronales de la región RRVMO, siendo la respuesta dependiente del sistema neuronal que resulta inhibido.

Acetilcolina

Muscarínicos (mAChR) nitotinicos (nAChR)

y

ación mAChR: A nivel de CPME (plexo colinérgico en lámina II) reduce el dolor y su inhibición por competencia con GABA induce hiperse nsibilidad nociceptiva.

Activación nAChR: A n ivel de los núcleos del tronco encefálico estimula las vías inhibitorias descendentes y actúa como un mediador de efectos nociceptivos de las vías adrenérgicas, serotoninérgicas y colinérgicas a nivel de ME.

Serotonina Antinociceptivos $\left(5-\mathrm{HT}_{1_{\mathrm{A}}}, \quad 5-\right.$ $\mathrm{HT}_{1 \mathrm{~B}}, \quad 5-\mathrm{HT}_{1 \mathrm{D}} \mathrm{y} \quad$ 5-HT 7 y Pronociceptivos $\left(5-\mathrm{HT}_{2} \mathrm{~A}\right.$ $\left.\mathrm{HT}_{3}\right)$

GABA

NMDA
Antinociocepción: La activación de proyecciones descendentes de la ME provoca la liberación de serotonina que inhibe a la primera neurona de transmisión del dolor debido a la hiperpolarización de los canales de $\mathrm{K}+$, lo que resulta en la inhibición de la liberación de neurotransmisores en la vía nociceptiva.

Pronociocepción: La regulación serotoninérgica ascendente aumenta el potencial de transmisión doloroso de las fibras C en el CPME.

La activación de los receptores NMDA y la apertura de los canales de calcio, desencadenan grandes flujos de iones de calcio, dependientes de la despolarización de la me mbrana. Como consecuencia ocurre liberación de GABA produciendo una inhibición generalizada en la transmisión nociceptiva en terminales sinápticas y extrasinápticas. 
c) Modulación del dolor mediada por Serotonina. A nivel de la región rostral ventromedial de la médula oblongada (RRVMO), las neuronas serotoninérgicas comprenden el 20 $\%$ del total de neuronas (Arbaiza; Ossipov et al., 2010) (Fig. 1). Las proyecciones serotoninégicas se distribuyen hacia el CPME y tienen su origen en los núcleos magno y reticular del rafe, las que representan una estructura intermedia entre el RRVMO y la porción medial del núcleo gigantocelular. La estimulación de estos núcleos produce analgesia y liberación de serotonina a nivel del CPME, mientras que la lesión de estos núcleos, disminuye el umbral doloroso y produce hiperalgesia (Perena et al.; Pedrajas \& Molino). Sin embargo, la modulación serotoninérgica participa tanto en procesos facilitadores como inhibidores en función del subtipo del receptor. En la Tabla II se entregan mayores detalles.

d) Aminoácidos inhibitorios que participan en la modulación del dolor. La colecistoquinina es una hormona gastrointestinal que se libera en respuesta a la ingesta alimentaria, sin embargo se han descubierto otros roles potenciales, entre los que se incluyen el dolor y la memoria. Por su parte, el ácido g-aminobutírico (GABA) es el neurotransmisor inhibitorio más predominante del SNC. La pérdida de la inhibición nociceptiva mediada por GABA resulta clave en el desarrollo de dolor inflamatorio y neuropático. En tanto, galanina es un neuropéptido capaz de participar tanto en la facilitación como en la inhibición de la nocicepción (Kirkpatrick et al., 2015).

En la Tabla II se resumen las sustancias neuromoduladoras del dolor más importantes, sus receptores y mecanismo de acción.

\section{Mecanismos moduladores corticales.}

La corteza cerebral es una de las principales áreas donde se produce la modulación nociceptiva. Esta, es capaz de mediar la respuesta inmediata ante un estímulo nocivo y la posterior experiencia afectiva. De manera similar, algunas áreas corticales pueden modular las reacciones motoras somáticas reflejas a un estímulo nocivo y participar en la organización de respuestas somáticas voluntarias. Por su parte, las respuestas autonómicas incluyen reacciones cardiorrespiratorias, vasomotoras y endocrinas, que están mediadas inmediatamente por mecanismos subcorticales, pero que pueden ser facilitadas o prolongadas por la acción de mecanismos corticales específicos (Cervero, 2012). Se ha postulado que el área prefrontal (APF) representa la fuente fundamental de modulación que inicia la actividad inhibitoria descendente y/o la modulación emocional del dolor. La ausencia de analgesia periférica se ha asociado con aumentos significativos de la señal cerebral en la APF y el área anterior del giro cingulado (AGC) (Ossipov et al.) (Fig. 1). Por otro lado, los estados emocionales pueden afectar la percepción del dolor y la magnitud de este efecto, lo que a menudo se correlaciona con alteraciones en la activación de la AGC. Es así como, a pesar de que el tronco encefálico contiene el circuito neural crítico para la modulación del dolor, este puede ser modificado por centros cerebrales más altos, estando significativamente influenciado por la ansiedad del individuo, la experiencia del dolor o incluso la expectativa analgésica, la que se ve reducida en individuos con dolor crónico (Navratilova et al., 2015; Naser \& Kuner).

\section{Mecanismos moduladores subcorticales.}

El núcleo accumbens (NAc) y el cuerpo amigdalino reciben información nociceptiva a través de proyecciones espinoparabraquial-amígdala (Fig. 1). El cuerpo amigdalino integra la información nociceptiva con el contenido afectivo, pudiendo ser modificada por emociones negativas y positivas, que a su vez pueden inhibir o facilitar el dolor. El núcleo central de la amígdala, establece conexiones directas e indirectas con sistemas de modulación descendentes, centrados en la red compuesta por la SGPA y la RRVMO (Cervero; Tobaldini et al., 2019). Existe evidencia acerca de la participación del cuerpo amigdalino en la inhibición endógena del dolor a través de la vía colinérgica, que le permite inducir un estado de analgesia contextual frente a estados de miedo o estrés y la facilitación del dolor mediante el GABA (Arbaiza; Cervero; Martins \& Tavares, 2017; Tobaldini et al.).

También se ha identificado la SGPA, que recibe información nociceptiva a través de los tractos espinoreticulares generando redes con la formación reticular (FR), los núcleos de rafe, el área parabraquial, el locus cerúleo (LC) y el hipotálamo (Bushnell et al., 2013; Chichorro et al.). La SGPA desempeña un papel fundamental en la transmisión de las influencias moduladoras de los centros cerebrales superiores involucrados en aspectos del dolor (Martins \& Tavares). LA SGPA presenta subdivisiones como el RRVMO, que proyecta vías adrenérgicas para el control autonómico (Arbaiza). La tríada RRVMO - médula espinal ventrolateral - proceso reticular espinal desempeñan un papel clave como puerta de entrada para permitir la modulación del dolor desde el cerebro, hacia la médula espinal. Se propone un circuito neuronal homeostático que permite reacciones corporales adecuadas en eventos potencialmente nocivos, como escapar de un depredador o un dolor agudo intenso. Estudios han demostrado la presencia de dos grupos celulares a nivel de RRVMO, las células-off y las células-on. Estas envían proyecciones hacia el CPME o al núcleo del tracto espinal trigeminal. Las células-on se activan previo al inicio del reflejo nociceptivo y están relacionadas con la facilitación 
del dolor. Las células-off disminuyen su actividad justo antes de finalizar el reflejo nociceptivo y son activadas por encefalinas en los receptores $\mu$ opioides, desenvolviéndose en aspectos inhibitorios del dolor. Las células-off, presentan abundantes ramificaciones dentro de la región, con efectos excitadores para otras células-off e inhibidores para las células-on. El sistema opioide inhibe al sistema de neuronas "on", y excita al sistema de neuronas "off" (Roeder et al., 2016; Martins \& Tavares).

Por su parte, el circuito hipotálamo lateral - área tegmental ventral del mesencéfalo - NAc tienen un rol no solo en el procesamiento de la recompensa frente al dolor sino también en la modulación de este, mediado por receptores dopaminérgicos (Martins \& Tavares; SiahposhtKhachaki et al., 2017; Shafiei et al., 2018). Respecto a la FR, esta se considera un sitio de relevo importante para la información nociceptiva ascendente en el tracto espinoreticulo-talámico. Se piensa que la FR esta involucrada en la mediación de los aspectos motivacionales y facilitación del dolor. Estas neuronas, tienen proyecciones descendentes hacia CPME y proyecciones ascendentes a la corteza frontal a través de un relevo en el tálamo (Cervero). Por otro lado, el LC, en sus áreas 5 y 7, presenta proyecciones noradrenérgicas que se dirigen hacia el CPME. La región A7 es un relevo crítico para la acción noradrenérgica espinal de la RRVMO, debido a que no solo se proyecta a esta región, sino que además recibe de ella proyecciones que contienen sustancia $\mathrm{P}$, la cual produce un efecto antinociceptivo a nivel espinal (Naser \& Kuner).

\section{Modulación a nivel del CPME.}

A nivel de las láminas superficiales del CPME existen interneuronas que se activan mediante vías descendentes de la SGPA y RRVMO, los cuales contienen neurotransmisores inhibitorios como GABA, glicina y encefalinas, sugiriendo que su liberación contribuye al control descendente del dolor (Kirkpatrick et al.). Los impulsos que se generan en la SGPA y la sustancia gris periventricular (SGPV) descienden hacia la RRVMO, desde donde se liberan endorfinas y encefalinas. Adicionalmente, a este nivel se generan impulsos que descienden a través del cordón espinal posterolateral de la médula espinal y terminan en la lámina II del CPME, donde se libera serotonina. La serotonina proveniente de las neuronas “off” de la RRVMO, conecta a través de una interneurona inhibidora que libera encefalina e inhibe la transmisión nociceptiva, modulando la neurona de proyección presináptica. A este nivel, se inhibe la primera neurona de transmisión del dolor debido a la hiperpolarización de los canales de $\mathrm{K}+$, lo que resulta en la inhibición de la liberación de neurotransmisores en la vía nociceptiva (Kirkpatrick et al.).
En la lámina II del CPME, es posible distinguir 2 tipos de interneuronas descritas por Cajal como células limitantes (CL) y células centrales (CC). Las CL reciben aferencias pronociceptivas, antinociceptivas y terminaciones descendentes que provienen de sistemas inhibidores del dolor, actuando como un filtro modulador al proyectar su axón hacia las neuronas de la lámina I. Por otro lado, las CC son células de naturaleza inhibitoria que poseen prolongaciones axonales y dendríticas que no suelen salir de la lamina II. Reciben aferencias primarias antinociceptivas, estableciendo contacto con dendritas de las neuronas espinotalámicas que recorren la lamina II (Kirkpatrick et $a l$. .). En situaciones de hiperalgesia mediada por estímulo de receptores ácido N-metil-D-aspártico (NMDA) a nivel del CPME, es posible observar un aumento en los genes que transcriben neurotransmisores del SNC tales como dinorfina y encefalina. Por otro lado, debido a que la RRVMO posee receptores distribuidos en todo el organismo, es posible que un estímulo nocivo active sistemas que provocan analgesia, como ocurre en las técnicas basadas en contrairritación. Para dar explicación a este fenómeno, se propuso el sistema de MCD.

\section{Modulación Condicionada de Dolor (MCD).}

El principio de la MCD se basa en el fenómeno de contrairritación, es decir, el dolor inhibe el dolor (Damien et al., 2018). La inhibición del dolor en un segmento del cuerpo podría modularse por la aplicación de un estímulo nocivo localizado, produciendo un efecto analgésico difuso (Porreca et al., 2002; Edwards et al., 2003; Ossipov et al.; Bannister et al., 2015; Damien et al.). Los mecanismos inhibitorios de la MCD se producen en el CPME o en los núcleos trigeminales al activar el sistema inhibidor descendente. Este sistema corresponde a una forma única de analgesia endógena, que integra la información proveniente de proyecciones espinales y establece comunicaciones con la SGPA, RRVMO, tálamo y cuerpo amigdalino, además de enviar proyecciones moduladoras del dolor hacia el CPME y núcleos del trigémino (Damien et al.). Las proyecciones neuronales serotoninérgicas y noradrenérgicas en estas regiones generan efectos inhibitorios y/o excitatorios en la modulación del dolor (Bannister et al., 2009; Bannister et al., 2015). Adicionalmente, la MCD es capaz de inhibir las neuronas a nivel de la lámina I del CPME, estructura que resulta fundamental para el procesamiento de signos relevantes para la homeostasis (Craig, 2003; Villanueva, 2009).

La MCD es la base para numerosos tipos de tratamientos cuyo objetivo es la modulación y activación del sistema inhibitorio descendente del dolor. Estos enfoques aplican estímulos periféricos dolorosos intensos, que reclutan aferencias nociceptivas (fibras $\mathrm{A} \delta$ y $\mathrm{C}$ ) y producen efectos 
analgésicos difusos (Damien et al.). La pérdida de la funcionalidad del sistema de MCD, puede manifestarse a través de la pérdida del control inhibitorio endógeno o el aumento de la facilitación del dolor. Estudios realizados en pacientes que presentan osteoartritis, fibromialgia o trastornos temporomandibulares, demostraron un aumento en la sensibilidad a estímulos nociceptivos, lo que indica inactividad del sistema MCD al ser comparado con el grupo control. Por lo tanto, se concluye que los cuadros de dolor crónico podrían asociarse con la desactivación o disregulación de estos sistemas (Lautenbacher et al., 2007; Arendt-Nielsen et al., 2010; Yarnitsky et al., 2012; Oono et al., 2014).

\section{CONCLUSIONES}

La neuromodulación del dolor se entiende como una matriz compleja que incluye regiones y neurotransmisores esenciales del SNC. Los principales neurotransmisores moduladores del dolor corresponden a opioides endógenos, acetilcolina, serotonina, GABA, entre otros, los que interconectan las distintas áreas neuronales involucradas en la modulación nociceptiva. Estas regiones, participan no solo en el procesamiento del dolor, sino también en la regulación autonómica y el manejo sensorial, cognitivo y emocional asociado. Dentro de los principales sistemas de modulación del dolor encontramos los mecanismos corticales, los subcorticales, del CPME y la MCD. Por lo tanto, conocer los circuitos, mecanismos y sustancias involucradas en la neuromodulación del dolor es fundamental para entender su fisiología y fisiopatología.

\section{AGRADECIMIENTOS}

Los autores quieren agradecer al Prof. Dr. Mariano del Sol Calderon por su apoyo en el uso de una correcta y actualizada Terminologia Anatomica.

WEN, S.; MUÑOZ, J.; MANCILLA, M.; BORNHARDT, T.; RIVEROS, A. \& ITURRIAGA, V. Mechanisms of Central Pain Modulation: Literature Review. Int. J. Morphol., 38(6):1803-1809, 2020 .

SUMMARY: Pain perception results from multiple and dynamic mechanisms in the central nervous system (CNS) and peripheral nervous system that inhibit or facilitate stimulation and nociceptive response. However, neuromodulation is mainly a function of the CNS. Nociceptive stimulus is detected by peripheral neurons receptors that synapse with the secondary afferent neurons of the spinal cord. These fibers cross to conform the ascending nociceptive pathways. Once the subcortical structures are reached, the thalamus`s neurons are activated; the thalamus send the stimulus to the somatosensory cortex, triggering the conscious perception of pain and activating the descending inhibitory system. For the nociceptive modulation to be carried out, the participation of various substances or neurotransmitters that connect specialized CNS areas is necessary. Therefore, the aim of this study was to review the literature regarding the mechanisms involved in central pain modulation processes.

KEY WORDS: Pain; Nociceptive pain; Chronic pain; Neurophysiology; Neuroanatomy; Neural Inhibition.

\section{REFERENCIAS BIBLIOGRÁFICAS}

Arbaiza, D. Neurofisiología del dolor. Bol. Dolor, 14(44):14-40, 2005.

Arendt-Nielsen, L.; Nie, H.; Laursen, M.; Laursen, B.; Madeleine, P.; Simonsen, O. \& Graven-Nielsen, T. Sensitization in patients with painful knee osteoarthritis. Pain, 149(3):573-81, 2010.

Bannister, K.; Bee, L. \& Dickenson, A. Preclinical and early clinical investigations related to monoaminergic pain modulation. Neurotherapeutics, 6(4):703-12, 2009.

Bannister, K.; Patel, R.; Gonçalves, L.; Townson, L. \& Dickenson, A. Diffuse noxious inhibitory controls and nerve injury: Restoring an imbalance between descending monoamine inhibitions and facilitations. Pain, 156(9):1083-11, 2015.

Bushnell, C.; Ceko, M. \& Low, L. Cognitive and emotional control of pain and its disruption in chronic pain. Nat. Rev. Neurosci., 14(7):502-11, 2013.

Cervero, F. Understanding Pain. Massachusetts. The MIT Press, 2012.

Chichorro, J.; Porreca, F. \& Sessle, B. Mechanisms of craniofacial pain. Cephalalgia, 37(7):613-26, 2017

Craig, A. A new view of pain as a homeostatic emotion. Trends Neurosci., 26(6):303-7, 2003.

Damien, J.; Colloca, L.; Bellei-Rodriguez, C. E. \& Marchand S. Pain modulation: from conditioned pain modulation to placebo and nocebo effects in experimental and clinical pain. Int. Rev. Neurobiol., 139:25596, 2018.

Edwards, R.; Ness, T.; Weigent, D. \& Fillingim, R. Individual differences in diffuse noxious inhibitory controls (DNIC): association with clinical variables. Pain, 106(3):427-37, 2003.

Kirkpatrick, D.; McEntire, D.; Hambsch, Z.; Kerfeld, M.; Smith, T.; Reisbig, M.; Youngblood, Ch. \& Agraval, D. Therapeutic Basis of Clinical Pain Modulation. Clin. Transl. Sci., 8(6):848-56, 2015.

Lautenbacher, S.; Prager, M. \& Rollman, G. B. Pain additivity, diffuse noxious inhibitory controls, and attention: a functional measurement analysis. Somatosens. Mot. Res., 24(4):189-201, 2007.

Martins, I. \& Tavares, I. Reticular formation and pain: The past and the future. Front. Neuroanat., 11:51, 2017.

Naser, P. V. \& Kuner, R. Molecular, cellular and circuit basis of cholinergic modulation of pain. Neuroscience, 387:135-48, 2018.

Navratilova, E.; Xie, J.; Meske. D.; Qu, C.; Morimura, K.; Okun, A.; Arakawa, N.; Ossipov, M.; Howard, L. \& Porreca, F. Endogenous opioid activity in the anterior cingulate cortex is required for relief of pain. $J$. Neurosci., 35(18):7264-71, 2015.

Okeson, J. Tratamiento de Oclusión y Afecciones Temporomandibulares. 7a ed. Barcelona, Elsevier, 2013.

Oono, Y.; Wang, K.; Baad-Hansen, L.; Futarmal, S.; Kohase, H.; Svensson, P. \& Arendt-Nielsen, L. Conditioned pain modulation in temporomandibular disorders (TMD) pain patients. Exp. Brain Res., 232(10):3111-9, 2014. 
Ossipov, M. H.; Dussor, G. O. \& Porreca, F. Central modulation of pain. J. Clin. Invest., 120(11):3779-87, 2010.

Pedrajas, J. \& Molino, Á. Bases neuromédicas del dolor. Clin. Salud, 19(3):277-93, 2008.

Perena, M. J.; Perena, M. F.; Rodrigo-Royo, M. \& Romera, E. Neuroanatomía del dolor. Rev. Soc. Esp. Dolor, 7(Supl. II):5-10, 2000.

Porreca, F.; Ossipov, M. \& Gebhart, G. Chronic pain and medullary descending facilitation. Trends Neurosci., 25(6):319-25, 2002.

Roeder, Z.; Chen, Q.; Davis, S.; Carlson, J.; Tupone, D. \& Heinricher, N. Parabrachial complex links pain transmission to descending pain modulation. Pain, 157(12):2697-708, 2016.

Shafiei, I.; Vatankhah, M.; Zarepour, L.; Ezzatpanah, S. \& Haghparast, A. Role of D1- and D2-like dopaminergic receptors in the nucleus accumbens in modulation of formalin-induced orofacial pain: Involvement of lateral hypothalamus. Physiol. Behav., 188:25-31, 2018.

Siahposht-Khachaki, A.; Pourreza, P.; Ezzatpanah, S. \& Haghparast, A. Nucleus accumbens dopamine receptors mediate hypothalamus-induced antinociception in the rat formalin test. Eur. J. Pain, 21(7):1285-94, 2017.

Tobaldini, G.; Sardi, N.; Guilhen, V. \& Fischer, L. Pain inhibits ain: an Aascending-descending pain modulation pathway linking mesolimbic and classical descending mechanisms. Mol. Neurobiol., 56(2):100013, 2019.

Villanueva, L. Diffuse noxious inhibitory control (DNIC) as a tool for exploring dysfunction of endogenous pain modulatory systems. Pain, 143(3):161-2, 2009.

Yarnitsky, D.; Granot, M.; Nahman-Averbuch, H.; Khamaisi, M. \& Granovsky, Y. Conditioned pain modulation predicts duloxetine efficacy in painful diabetic neuropathy. Pain, 153(6):1193-8, 2012.

Zegarra, J. Bases fisiopatológicas del dolor. Acta Med. Peru., 24(2):35-8, 2007.

\author{
Dirección para correspondencia: \\ Prof. Veronica Iturriaga \\ Departamento de Odontología Integral Adulto \\ Facultad de Odontología \\ Universidad de La Frontera \\ Avenida Francisco Salazar 01145 \\ Temuco \\ CHILE
}

Email: veronica.iturriaga@ufrontera.cl

Recibido : 08-06-2020

Aceptado: 11-08-2020 